\title{
Predicting pathological promoter-enhancer rewiring in chromosomal rearrangements
}

\author{
V. Fishman ${ }^{1,2 *}$, P. Belokopytova ${ }^{1,2}$, P. Salnikov ${ }^{1,2}$, D. Fishman ${ }^{2}$ \\ ${ }^{1}$ Institute of Cytology and Genetics SB RAS, Novosibirsk, Russia \\ ${ }^{2}$ Novosibirsk State University, Novosibirsk, Russia \\ *e-mail:minja@bionet.nsc.ru
}

Key words: genome architecture, chromosomal rearrangements, promoters, enhancers, Hi-C

Motivation and Aim: in mammals transcription of genes is precisely regulated by multiple molecular mechanisms. A crucial part of regulatory process is specific pairing of promoters and enhancers. Despite recent progress in genome-wide identification of enhancer sequences, it is not clear what determine specificity of 3-dimentional interactions between regulatory elements. Recently, several computational algorithms were employed to predict spatial interactions based on epigenetic signatures of promoters, enhancers and surrounding genomic regions. Here, we aimed to extend these algorithms to explain or predict alterations of 3-dimentional organization of chromatin and accompanying changes of gene expression, induced by chromosomal rearrangements.

Methods and Algorithms: We modify recently published machine-learning algorithm [1] to predict promoter-enchanter interactions in mouse and human genomes. To train algorithm and estimate importance of predictors, we used known promoter-enhancer interactions from [2]. Next, we used the trained model to predict gain or loss of interactions in genome altered by chromosomal rearrangement. We utilized publically available mouse (IMPC, Jaxon Lab) and human (OMIM, ClinVar) databases to suggest phenotypical consequences associated with promoter-enhancer rewiring caused by chromosomal rearrangement. Finally, we used CRISPR/Cas9 tools to establish experimental system for validation of model prediction.

Results and Conclusion: We developed a machine learning algorithm to predict loss or acquirement of promoter-enhancer interactions caused by chromosomal rearrangements. We estimated impact of different biological features on prediction accuracy and suggested the optimal set of features. We used the algorithm to predict promoter-enhancer rewiring caused by defined chromosomal rearrangements. Finally, we showed that CRISPR/Cas9 system can be utilized to validate prediction of developed algorithm.

Acknowledgements: This study was supported by the RSF (grant 17-74-10143).

\section{References}

1. Whalen S. et al. (2016) Enhancer-promoter interactions are encoded by complex genomic signatures on looping chromatin. Nat. Genet. 48(5):488-496.

2. Shen Y. et al. (2012) A map of the cis-regulatory sequences in the mouse genome. Nature. 488(7409): 116-120. 